\title{
Uniqueness and Nonuniqueness in Inverse Problems for Elliptic Partial Differential Equations and Related Medical Imaging
}

\author{
Kiwoon Kwon \\ Department of Mathematics, Dongguk University, Seoul 100-715, Republic of Korea \\ Correspondence should be addressed to Kiwoon Kwon; kwkwon@dongguk.edu
}

Received 12 November 2014; Accepted 12 January 2015

Academic Editor: Hagen Neidhardt

Copyright (C) 2015 Kiwoon Kwon. This is an open access article distributed under the Creative Commons Attribution License, which permits unrestricted use, distribution, and reproduction in any medium, provided the original work is properly cited.

Unique determination issues about inverse problems for elliptic partial differential equations in divergence form are summarized and discussed. The inverse problems include medical imaging problems including electrical impedance tomography (EIT), diffuse optical tomography (DOT), and inverse scattering problem (ISP) which is an elliptic inverse problem closely related with DOT and EIT. If the coefficient inside the divergence is isotropic, many uniqueness results are known. However, it is known that inverse problem with anisotropic coefficients has many possible coefficients giving the same measured data for the inverse problem. For anisotropic coefficient with anomaly with or without jumps from known or unknown background, nonuniqueness of the inverse problems is discussed and the relation to cloaking or illusion of the anomaly is explained. The uniqueness and nonuniqueness issues are discussed firstly for EIT and secondly for ISP in similar arguments. Arguing the relation between source-to-detector map and Dirichlet-to-Neumann map in DOT and the uniqueness and nonuniqueness of DOT are also explained.

\section{Introduction}

Let us consider the following second order elliptic partial differential equations with Diriclet boundary value in a Lipschitz domain $\Omega$ compactly embedded in $\mathbb{R}^{n}$ :

$$
\begin{gathered}
-\nabla \cdot(\sigma \nabla u)+k^{2} \tau u=q \quad \text { in } \Omega, \\
u=f \quad \text { on } \partial \Omega,
\end{gathered}
$$

where $k$ is a nonnegative number, $q, f$ are real functions satisfying $q \in H^{-1}(\Omega), f \in H^{1 / 2}(\partial \Omega)$, and $\sigma$ is a real matrix and $\tau$ is a nonnegative function such that

$$
L \leq \frac{y^{t} \sigma y}{y^{t} y} \leq U, \quad 0 \leq \tau \leq U
$$

for all $y \in \mathbb{R}^{n} \backslash\{\mathbb{O}\}$ and some positive constants $L$ and $U$. It is known that (la) and (1b) have a unique solution $u \in H^{1}(\Omega)$ [1]. Therefore, we can define the Dirichlet-to-Neumann map $\Lambda_{\sigma, \tau}: H^{1 / 2}(\partial \Omega) \rightarrow H^{-1 / 2}(\partial \Omega)$ as $\Lambda_{\sigma, \tau}(f)=\left.\nu \cdot(\sigma \nabla u)\right|_{\partial \Omega}$ using the boundary trace operator. We will explain EIT, ISP, and DOT using (1a) and (1b) and Dirichlet-to-Neumann map or corresponding measurements map.
When $k=q=0$, EIT is formulated as to find $\sigma$ such that $\Lambda_{\sigma}\left(:=\Lambda_{\sigma, 0}\right)=\Lambda$ for given Dirichlet-to-Neumann map $\Lambda$. In finite measurements case, EIT is to find the conductivity $\sigma$ satisfying $\Lambda_{\sigma}\left(f_{i}\right)=\Lambda\left(f_{i}\right), i=1, \ldots, N$, for given finite Dirichlet and Neumann boundary measurement pairs $\left(f_{i}, \Lambda\left(f_{i}\right)\right)_{i=1, \ldots, N}$.

We discuss uniqueness of nonuniqueness of EIT by classifying $\sigma$ into six cases for a Lipschitz domain $D$ compactly embedded in $\Omega$ such that $\Omega \backslash \bar{D}$ is connected:

$$
\begin{aligned}
& \text { Case 1: } \sigma=\chi_{\Omega \mid \bar{D}}+b \chi_{D}, b \neq 1 \text { on } \partial D, \\
& \text { Case 2: } \sigma=b(x), \\
& \text { Case 3: } \sigma=\chi_{\Omega \mid \bar{D}}+b(x) \chi_{D}, b(x) \neq 1 \text { on } \partial D, \\
& \text { Case 4: } \sigma=I_{n} \chi_{\Omega \mid \bar{D}}+B \chi_{D}, B \neq I_{n} \text { on } \partial D, \\
& \text { Case 5: } \sigma=B(x), \\
& \text { Case 6: } \sigma=I_{n} \chi_{\Omega \mid \bar{D}}+B(x) \chi_{D}, B(x) \neq I_{n} \text { on } \partial D,
\end{aligned}
$$

where

(i) $b$ : a positive number,

(ii) $b(x)$ : a positive function, 
(iii) $B$ : a symmetric positive-definite matrix,

(iv) $B(x)$ : a symmetric positive-definite matrix function,

(v) $I_{n}$ : the $n \times n$ identity matrix.

For Cases 1,2,3, and 4, uniqueness of the coefficient $\sigma$ is known for general conditions on regularity of the conductivity. For Case 5, cloaking is heavily studied recently. Invisibility and cloaking of $D$ are closely related with the nonuniqueness of coefficients not only in EIT [2-6], but also in acoustic scattering [7-9], electromagnetic scattering [10-12], and quantum scattering [13]. The idea of physical devices related to cloaking or invisibility is suggested more concretely than before such as wormhole and metamaterials and these studies draws much attention from physics and material engineering societies [14-16]. However, in Case 6, cloaking of the domain of anomaly $D$ is impossible but the illusion of the property $B(x)$ is possible as in [11].

Acoustic wave propagation is described as follows:

$$
\begin{gathered}
\nabla \cdot(\sigma \nabla u)+k^{2} \tau u=0 \quad \text { in } \mathbb{R}^{n}(n=2,3) \\
u(x)=e^{i k x \cdot d}+u^{s}(x) \\
\lim _{r \rightarrow \infty} r^{(n-1) / 2}\left(\frac{\partial u^{s}}{\partial r}(r \hat{x})-i k u^{s}(r \hat{x})\right)=0 \\
\text { uniformly for all } \hat{x}=\frac{x}{r} \in S^{n-1},
\end{gathered}
$$

where $\sigma$ is a positive-definite matrix function, $\tau$ is a positive function, and

$$
\sigma-I_{n}, \quad \tau-1 \text { have compact supports. }
$$

It is known that there exists a unique function $u^{s}$ satisfying (3) and (4) [17]. $u^{s}$ is called scattering wave. The scattering wave $u^{s}$ is approximated by far-field pattern $u_{\infty}$ by the following relation:

$$
u^{s}(x)=r^{(1-n) / 2} e^{i k r}\left(u_{\infty}(\widehat{x})+O\left(\frac{1}{r}\right)\right) .
$$

The inverse scattering problem (ISP) is defined as follows: given far-field patterns $u_{\infty}(\cdot, d)$ for all incident directions $d \epsilon$ $S^{n-1}, n=2,3, \ldots$, identify coefficients $\sigma$ and $\tau$.

In this paper, we classify $\sigma$ and $\tau$ as the following cases:

$$
\begin{aligned}
& \text { Case 7: } \sigma=1, \tau=\chi_{\mathbb{R}^{n} \backslash \bar{D}}+c \chi_{D}, c \neq 1 \text { on } \partial D, \\
& \text { Case 8: } \sigma=1, \tau=\chi_{\mathbb{R}^{n} \backslash \bar{D}}+c(x) \chi_{D}, c(x) \neq 1 \text { on } \partial D, \\
& \text { Case 9: } \sigma=1, \tau=c(x), \\
& \text { Case 10: } \sigma=I_{n} \chi_{\mathbb{R}^{n} \backslash \bar{\Omega}}+B_{+}(x) \chi_{\Omega \backslash \bar{D}}+B_{-}(x) \chi_{D}, \tau= \\
& \chi_{\mathbb{R}^{n} \backslash \bar{D}}+c(x) \chi_{D}, \\
& \text { Case 11: } \sigma=I_{n} \chi_{\mathbb{R}^{n} \backslash \bar{D}}+B(x) \chi_{D}, \tau=\chi_{\mathbb{R}^{n} \backslash \bar{D}}+ \\
& c(x) \chi_{D}, c(x) \neq 1, B(x) \neq I_{n} \text { on } \partial D,
\end{aligned}
$$

where $c$ is a complex number and $c(x)$ is a complex function. Note that from (2) we have

$$
L \leq \frac{y^{t} B(x) y}{y^{t} y}, c, c(x) \leq U
$$

for all $y \in \mathbb{R}^{n} \backslash\{\mathbb{O}\}$.
ISP for (4) with $\sigma, \tau$ given in Cases $7,8,9,10$, and 11 is equivalently formulated as an inverse problem to find $\sigma$ and $\tau$ from $\Lambda_{\sigma, \tau}$ in (1a) and (1b) for some Lipschitz domain $\Omega$ compactly embedded in $\mathbb{R}^{n}$ and containing $\bar{D}$ and $q=0$. By Theorem 6.1.3 in [18], we can take $\Omega$ as a ball centered at the origin with radius $R$ being chosen such that $k$ is not a Dirichlet eigenvalue of (1a) with $q=0$.

In this paper, DOT is explained as an inverse problem with respect to a forward problem formulated as an elliptic partial differential equation. Propagation of light in biological tissues is usually described by diffusion approximation equation in the frequency domain, the simplest but nontrivial approximation of the Boltzmann equation, as follows:

$$
\begin{gathered}
-\nabla \cdot(\kappa \nabla \Phi)+\left(\mu_{a}+\frac{i m \omega}{l}\right) \Phi=q \quad \text { in } \Omega \\
\Phi+2 a \nu \cdot(\kappa \nabla \Phi)=0 \quad \text { on } \partial \Omega,
\end{gathered}
$$

where $\Phi$ is photon density distribution, $\mu_{a}$ absorption coefficient, $\mu_{s}^{\prime}$ reduced scattering coefficient, $\kappa=1 / 3\left(\mu_{a}+\mu_{s}^{\prime}\right)$ a diffusion coefficient, and $m$ refractive index. Usually, we assume $m=1$ and $a$ is boundary reflection coefficient, $l$ the speed of light, $\omega$ modulation frequency of light, and $v$ outer unit normal vector.

DOT is to find the optical coefficients $\mu_{a}$ and $\mu_{s}^{\prime}$ from the measurement informations $\Phi_{i, j}$ which is the value of the solution of (7a) and (7b) at $r_{i} \in \partial \Omega$ when $q(r)=\delta\left(r, r_{j}\right), r_{j} \in$ $\partial \Omega$. The $r_{i}$ and $r_{j}$ are usually called source and detector point, respectively.

Near infrared light is known to be deepest in the penetration depth to the tissue, compared to visible or nearvisible lights. Therefore, near infrared light is used in DOT. DOT is known to be of low cost, portable, nonionized, and nonmagnetized. And DOT has higher temporal resolution and more functional information than conventionl structural medical imaging modalities such as magnetic resonance imaging (MRI) and computerized tomography (CT). For the comparison to other functional imaging modalities such as functional MRI (fMRI), photon emission tomography (PET), and electroencephalogram (EEG), see [19]. DOT is used in the area of breast imaging [20-22], functional neuroimaging $[23,24]$, brain computer interface (BCI) $[25,26]$, and the study about seizure [27, 28], new born infants [29, 30], osteoarthritis [31], and rat brain [32, 33].

We interpret DOT also as an inverse problem for (1a) and (lb) in isotropic coefficient and the uniqueness is discussed in the following cases:

Case 12: $m=m_{0}$ is given and $\omega \neq 0$, space dimension $n \geq 3$,

Case 13: $m=m_{0}$ is given and $\omega \neq 0$, space dimension $n=2$,

Case 14: $\omega=0$ or $n$ is to be determined.

In Sections 2, 3, and 4, uniqueness and nonuniqueness of EIT, ISP, and DOT are treated, respectively. The relation between nonuniqueness and cloaking or illusion is also explained. 


\section{EIT}

Consider $k=q=0$ for the Dirichlet elliptic problem (1a) and (1b). EIT is formulated as follows:

(i) find $\sigma$ such that $\Lambda_{\sigma}\left(:=\Lambda_{\sigma, 0}\right)=\Lambda$ for given or measured Dirichlet-to-Neumann map $\Lambda$.

2.1. Uniqueness in Cases 1,2, 3, and 4. The uniqueness studies of EIT in Cases 1,2,3, and 4 are summarized in the following way.

Case 1. One or two measurements uniqueness results are known when $b$ is assumed to be known [34-36]. The finite measurement uniqueness is known only for Case 1. In this case, the number of measurements, the geometry of the obstacle $D$, and the choice of suitable Dirichlet or Neumann data minimizing the number of measurements are interesting issues.

Case 2. Many mathematicians conduct extensive works in this case and thus we are able to understand the unique determination of electrical conductivity when $b(x) \in L^{\infty}(\Omega)$ for two dimensional case [37-39] and $b(x) \in C^{3 / 2}(\Omega)$ for the case in dimensions higher than two [40-43].

Case 3. In [44], an orthogonality relation between the two solutions of (7a) and (7b) for arbitary obstacles $D_{1}$ and $D_{2}$ is derived. Based on this orthogonal relation and the HahnBanach theorem, the uniqueness for Case 3 is derived.

Case 4. The uniqueness in Case 4 is also proved in [44] with additional condition that $B-A$ is positive-definite: this additional condition is generalized and removed by $[45,46]$.

2.2. Nonuniqueness in Case 5. The nonuniqueness of EIT in Case 5 is observed early in [47]: if $F$ is a boundary fixing diffeomorphism on $\Omega$ and push-forward map $F_{*}$ is defined by

$$
F_{*} \sigma=\frac{(D F) \sigma(D F)^{t}}{\operatorname{det}(D F)},
$$

then we have

$$
\Lambda_{\sigma}=\Lambda_{F_{*} \sigma} .
$$

The proof of (9) is summarized well in [6]: Knowing the Dirichlet-to-Neumann map is equivalent to knowing the quadratic form

$$
Q(v)=\int \sigma \nabla v \cdot \nabla v \quad v \in H^{1}(\Omega)
$$

by the polarization identity. Then, (9) follows from the change of variable method for the quadratic form

$$
\begin{aligned}
& \int_{\Omega} \sum_{i j} \sigma_{i j} \frac{\partial u}{\partial x_{i}} \frac{\partial u}{\partial x_{j}} d x \\
& =\int_{\Omega} \sum_{i j} \sigma_{i j} \frac{\partial u}{\partial y_{k}} \frac{\partial y_{k}}{\partial x_{i}} \frac{\partial u}{\partial y_{l}} \frac{\partial y_{l}}{\partial x_{j}} \operatorname{det}\left(\frac{\partial x}{\partial y}\right) d y .
\end{aligned}
$$

Many researchers raised questions whether the change of variable (9) is a unique obstruction to the uniqueness. And it is proved that

$$
\Lambda_{\sigma_{1}}=\Lambda_{\sigma_{2}} \text { implies } \sigma_{2}=F_{*} \sigma_{1}
$$

for some boundary fixing diffeomorphism $F$ in the following cases:
(i) $n=2$ and $\sigma \in C^{3}(\Omega)[48]$,
(ii) $n=2$ and $\sigma \in C^{0,1}(\Omega)$ (Lipschitz functions) [49],
(iii) $n=2$ and $\sigma \in L^{\infty}(\Omega)[50]$,
(iv) $n \geq 3: \partial D$ and $B$ are analytic $[51,52]$.

2.3. Near-Cloaking in Case 5. Let $D$ be some domain and contained in $\Omega, B_{+}=\left.B\right|_{\Omega \backslash \bar{D}}, B_{-}=\left.B\right|_{D}$, and

$$
\sigma=B_{+}(x) \chi_{\Omega \backslash \bar{D}}+B_{-}(x) \chi_{D} .
$$

Define

$$
\sigma_{F}:=F_{*} \sigma=I_{n} \chi_{\Omega \backslash \epsilon \bar{D}}+F_{*} B_{-}(x) \chi_{\epsilon D}(x),
$$

for a boundary-fixing diffeomorphism $F$ on $\Omega$ transforming $D$ into arbitrary small domain $\epsilon D$ and $F_{*} B_{+}=I_{n}$ on $\Omega \backslash \bar{D}$. Then, $D$ is nearly cloaked into $\epsilon D$, since $\Lambda_{\sigma}=\Lambda_{\sigma_{F}}$ by (9). If $\epsilon$ goes to $0, D$ is called perfectly cloaked. Otherwise, $D$ is called nearly cloaked into $\epsilon D$. Conversely, if $D$ is nearly cloaked into $\epsilon D$, there is a diffeomorphism $F$ such that $\Lambda_{\sigma}=\Lambda_{\sigma_{F}}$ by (12).

For example, if $\Omega, D$ and $\epsilon D$ are two-dimensional disks of radii 2,1 , and $\epsilon>0$, respectively, centered at $\mathbb{O}$, we could take the diffeomorphism $F$ on $\Omega$ mapping $D$ into $\epsilon D$ as follows:

$$
F(x)=\epsilon x \chi_{D}(x)+((2-\epsilon)|x|-(2-2 \epsilon)) \frac{x}{|x|} \chi_{\Omega \backslash \bar{D}}(x) .
$$

2.4. Illusion in Case 6. In Case 6, the uniqueness of $D$ is solved in $[44,45,53]$ and the nonuniqueness of $B(x)$ inside $D$ is shown using (12) [53]. This is called illusion of material property $B(x)$ inside the uniquely determined domain $D$. Note that $D$ is not cloaked into $\epsilon D$ any more.

In more detail, if

$$
\Lambda_{I_{n} \chi_{\Omega \mid \bar{D}_{1}}+B_{1}(x) \chi_{D_{1}}}=\Lambda_{I_{n} \chi_{\Omega \backslash \bar{D}_{2}}+B_{2}(x) \chi_{D_{2}}}
$$

then we have

$$
D_{1}=D_{2}
$$

And there is a boundary fixing diffeomorphism $F$ on $D$ such that

$$
B_{2}=F_{*} B_{1} \text {. }
$$

Therefore, in Case 6, the domain $D$ is uniquely determined by the Dirchlet-to-Neumann map; however, the property $B$ is nonunique up to the change of variables inside $D_{1}=$ $D_{2}$. Specifically, the domain of anomaly $D$ cannot be cloaked into a much smaller domain, but the property $B_{1}$ could be illuded into other property $B_{2}$. We will call (18) property illusion or just illusion. 
2.5. Cloaking in Case 5 versus Illusion in Case 6. Define three kinds of conductivities as follows:

$$
\begin{aligned}
& \sigma_{1}=I_{n} \chi_{\Omega \backslash \bar{D}}+B_{1} \chi_{D}, \\
& \sigma_{2}=B_{2+} \chi_{\Omega \backslash \bar{D}}+B_{2-} \chi_{D}, \\
& \sigma_{3}=I_{n} \chi_{\Omega \backslash \epsilon \bar{D}}+B_{3} \chi_{\epsilon D} .
\end{aligned}
$$

Cloaking and illusion for three conductivities (19a), (19b), and $(19 \mathrm{c})$ are summarized as follows:

(i) $\Lambda_{\sigma_{1}}=\Lambda_{\sigma_{3}}$ implies that $\epsilon=1$ and, with some more assumptions [53], there exists a diffeomorphism $F$ on $D$ such that $\sigma_{1}=F_{*} \sigma_{3}$. Therefore, only property illusion between $B_{1}$ and $B_{3}$ is possible and near cloaking of domain is not possible;

(ii) $\Lambda_{\sigma_{2}}=\Lambda_{\sigma_{3}}$ implies that there exists a diffeomorphism $F$ on $\Omega$ such that $\sigma_{2}=F_{*} \sigma_{3}$. Therefore, near-cloaking of domain is possible.

Comparing the above two results, we can conclude that if background conductivity is known only on the boundary, near-cloaking of the domain $D$ is possible to any smaller domain $\epsilon D$. But if the background conductivity is known in the neighborhood of the boundary, near-cloaking of the domain $D$ is not possible and only property illusion between $B_{1}$ and $B_{2}$ is possible.

\section{ISP}

ISP is formulated as follows:

(i) given far-field patterns $u_{\infty}(\cdot, d)$ for all incident directions $d \in S^{n-1}, n=2,3, \ldots$, identify coefficients $\sigma$ and $\tau$ for (4).

Let $E_{R}$ be a ball centered at the origin with radius $R$ being chosen such that $k$ is not a Dirichlet eigenvalue of (1a) with $q=0$ and compactly embedded in $\mathbb{R}^{n}$ and containing $\bar{D}$. Then ISP is also formulated as follows (Theorem 6.1.3 in [54]):

(ii) find $\sigma$ and $\tau$ from $\Lambda_{\sigma, \tau}$ in (1a) and (1b) with $q=0$ for $E_{R}$.

\subsection{Uniqueness in Cases 7, 8, and 9}

Case 7. The case for positive constant $c$ can be understood as special cases of Case 8. The limiting cases $c=\infty$ and $c=0$ could be considered as (3) in $\Omega \backslash \bar{D}$ and the boundary condition on $\partial D$ as $\Phi=0$ (sound-soft case) and $\partial \Phi / \partial \nu=0$ (sound-hard case) on $\partial D$. The uniqueness for sound-soft and sound-hard obstacle $D$ is considered in $[17,55]$.

Case 8. This case is called "inverse transmission problem" and the uniqueness is solved in [17].

Case 9. Uniqueness with a few subcases is solved in [18].
3.2. Nonuniqueness in Case 10. Let $F: \mathbb{R}^{n} \rightarrow \mathbb{R}^{n}$ be a diffeomorphism such that $\left.F\right|_{\mathbb{R}^{n} \backslash \Omega}$ is an identity map on $\mathbb{R}^{n} \backslash \Omega$. Then

$$
u_{\sigma, \tau}^{\infty}(\cdot, d)=u_{F_{*}(\sigma, \tau)}^{\infty}(\cdot, d) \quad \forall d \in S^{n-1},
$$

where push-forward map is defined by

$$
F_{*} \sigma=\frac{(D F) \sigma(D F)^{t}}{\operatorname{det}(D F)}, \quad F_{*} \tau=\frac{\tau}{\operatorname{det}(D F)} .
$$

This is just change of variable in the weak formulation of the direct problem such that

$$
\begin{aligned}
& \int_{\Omega} \sum_{i j} \sigma_{i j} \frac{\partial u}{\partial x_{i}} \frac{\partial u}{\partial x_{j}} d x \\
& =\int_{\Omega} \sum_{i j} \sigma_{i j} \frac{\partial u}{\partial y_{k}} \frac{\partial y_{k}}{\partial x_{i}} \frac{\partial u}{\partial y_{l}} \frac{\partial y_{l}}{\partial x_{j}} \operatorname{det}\left(\frac{\partial x}{\partial y}\right) d y, \\
& \int_{\Omega} \tau u^{2} d x=\int_{D} \tau u^{2} \operatorname{det}\left(\frac{\partial x}{\partial y}\right) d y .
\end{aligned}
$$

We have similar question for EIT. Is the change of variable unique obstruction to the uniqueness of anisotropic ISP? And it is proved that

$$
u_{\sigma_{1}}^{\infty}(\cdot, d)=u_{\sigma_{2}}^{\infty}(\cdot, d) \quad \forall d \in S^{n-1} \text { implies } \sigma_{2}=F_{*} \sigma_{1}
$$

for some boundary fixing diffeomorphism $F$ in the following references:

$$
\begin{aligned}
& \text { (i) } n=2 \text { [56], } \\
& \text { (ii) } n \geq 3 \text { [57]. }
\end{aligned}
$$

3.3. Near-Cloaking in Case 10. Let $D$ be some domain and contained in $\Omega, B_{+}=\left.B\right|_{\Omega \mid \bar{D}}, B_{-}=\left.B\right|_{D}$, and

$$
\sigma=I_{n} \chi_{\mathbb{R}^{n} \mid \bar{\Omega}}+B_{+}(x) \chi_{\Omega \mid \bar{D}}+B_{-}(x) \chi_{D} .
$$

Define

$$
\sigma_{F}:=F_{*} \sigma=I_{n} \chi_{\mathbb{R}^{n} \mid \epsilon \bar{D}}+F_{*} B_{-}(x) \chi_{\epsilon D}(x),
$$

for a diffeomorphism $F$ on $\mathbb{R}^{n}$ transforming $D$ into arbitrary small domain $\epsilon D, F_{*} B_{+}=I_{n}$ on $\Omega \backslash \bar{D}$, and fixing $\mathbb{R}^{n} \backslash \bar{\Omega}$. Then, $D$ is nearly cloaked into $\epsilon D$, since $u_{\sigma}(\cdot, d)=u_{\sigma_{F}}^{\infty}(\cdot, d)$ for all $d \in S^{n-1}$ by (20). If $\epsilon$ goes to $0, D$ is called perfectly cloaked. Otherwise, $D$ is called nearly cloaked into $\epsilon D$. Conversely, if $D$ is nearly cloaked into $\epsilon D$, there is a diffeomorphism $F$ such that $u_{\sigma}(\cdot, d)=u_{\sigma_{F}}^{\infty}(\cdot, d)$ for all $d \in S^{n-1}$ by $(23)$.

For example, if $\Omega, D$ and $\epsilon D$ are two-dimensional disks of radii 2,1 , and $\epsilon>0$, respectively, centered at $\mathbb{O}$, we could take the diffeomorphism $F$ on $\Omega$ mapping $D$ into $\epsilon D$ as follows:

$$
\begin{aligned}
F(x)= & \epsilon x \chi_{D}(x)+((2-\epsilon)|x|-(2-2 \epsilon)) \frac{x}{|x|} \chi_{\Omega \mid \bar{D}}(x) \\
& +\chi_{\mathbb{R}^{n} \mid \bar{\Omega}}(x) .
\end{aligned}
$$


3.4. Illusion in Case 11. In case $11, D$ is uniquely determined and is studied in $[18,53,58-60]$ but $B(x)$ is not uniquely determined. That is to say, $D$ is uniquely determined and not cloaked into any smaller domain but $B(x)$ is illuded into another property $B^{\prime}$ by some diffeomorphism $F$ on $\mathbb{R}^{n}$. In more detail, if

$$
u_{\sigma_{1}, \tau_{1}}^{\infty}(\cdot, d)=u_{\sigma_{2}, \tau_{2}}^{\infty}(\cdot, d) \quad \forall d \in S^{n-1},
$$

where

$$
\begin{array}{r}
\sigma_{i}=I_{n} \chi_{\mathbb{R}^{n} \backslash D_{i}}+B_{i}(x) \chi_{D_{i}}, \\
\tau_{i}=\chi_{\mathbb{R}^{n} \backslash D_{i}}+c(x) \chi_{D_{i}}, \\
i=1,2,
\end{array}
$$

then we have

$$
D_{1}=D_{2}
$$

and there is exterior fixing diffeomorphism $F$ on $D$ such that

$$
B_{2}=F_{*} B_{1}, \quad c_{2}=\frac{c_{1}}{|\operatorname{det}(D F)|} .
$$

In [11], a few interesting property illusions are considered; the optical transformation of an object into another object with different property is considered. In the paper, stereoscopic image of a man is transformed into an illusion image of a woman and dielectric spoon of the electric permeability 2 into an illusion image of metallic cup of electric permeability -1 in an electromagnetic scattering problem.

3.5. Cloaking in Case 10 and Illusion in Case 11. Define three pairs of coefficients as follows:

$$
\begin{aligned}
& \left(\sigma_{1}(x), q_{1}(x)\right) \\
& \quad=\chi_{\mathbb{R}^{n} \backslash \bar{D}}(x)+\left(B_{1}(x), c_{1}(x)\right) \chi_{D}(x) \\
& \left(\sigma_{2}(x), q_{2}(x)\right) \\
& \quad=\chi_{\mathbb{R}^{n} \backslash \bar{\Omega}}(x)+\left(B_{2+}, c_{2+}\right) \chi_{\Omega \backslash \bar{D}}(x)+\left(B_{2-}, c_{2-}\right) \chi_{D}(x) \\
& \left(\sigma_{3}(x), q_{3}(x)\right) \\
& \quad=\chi_{\mathbb{R}^{n} \backslash \overline{\epsilon D}}(x)+\left(B_{3}(x), c_{3}(x)\right) \chi_{\epsilon D}(x)
\end{aligned}
$$

where $D$ is compactly imbedded in $\Omega$, which is also compactly imbedded in $\mathbb{R}^{n}$.

Let us denote $u_{i}^{\infty}:=u_{\sigma_{i}, \tau_{i}}^{\infty}$. Cloaking and illusion for three coefficients in (31a), (31b), and (31c) are summarized as follows:

(i) $u_{1}^{\infty}(\cdot, d)=u_{2}^{\infty}(\cdot, d)$ for all $d \in S^{n-1}$ implies $\epsilon=1$ and, with additional assumptions in [53], there is a exterior fixing diffeomorphism $F$ on $D$ such that

$$
\left(B_{3}(x), c_{3}(x)\right)=F_{*}\left(B_{1}(x), c_{1}(x)\right)
$$

$[56,57]$. Therefore, only property illusion between $B_{1}$ and $B_{2}$ is possible and near-cloaking of domain $D$ is not possible:

(ii) $u_{2}^{\infty}(\cdot, d)=u_{3}^{\infty}(\cdot, d)$ for all $d \in S^{n-1}$ implies that there is an exterior fixing diffeomorphism $F$ on $D$ such that

$$
\left(B_{3}(x), c_{3}(x)\right)=F_{*}\left(B_{2}(x), c_{2}(x)\right) .
$$

Therefore, near-cloaking of the domain is possible in this case.

Also as in EIT, we can conclude that if background identity coefficient is known only in $\mathbb{R}^{n} \backslash \Omega$, near-cloaking of the domain is possible to any smaller domain $\epsilon D$. But if the background coefficient is known and fixed in $\mathbb{R}^{n} \backslash D$, near-cloaking of the domain is not possible and only property illusion between $B_{1}$ and $B_{2}$ is possible.

\section{DOT}

DOT is formulated using source-to-detector map as follows:

(i) find the optical coefficients $\mu_{a}$ and $\mu_{s}^{\prime}$ from the measurement informations $\Phi_{i, j}$ which is the value of the solution of (7a) and (7b) at $r_{i} \in \partial \Omega$ when $q(r)=$ $\delta\left(r, r_{j}\right), r_{j} \in \partial \Omega$.

$r_{i}$ and $r_{j}$ are usually called source and detector point, respectively. We assumed that $\mu_{a}, \mu_{s}^{\prime}$ are isotropic in this paper.

Suppose that $\partial \kappa / \partial \nu=0$ on $\partial \Omega$. Then DOT is also formulated using Dirichlet-to-Neumann map as follows:

(ii) find complex valued $\tau$ from $\Lambda_{1, \tau}$ in (1a) and (1b) with $q=0$ and given $k=1$.

4.1. Source-to-Detector Map, Dirichlet-to-Neumann Map, and Far-Field Map. We summarize the relation between sourceto-detector map and Dirichlet-to-Neumann map following the approach used in [61].

$$
\begin{gathered}
\text { By setting } \Psi= \\
\sqrt{\Delta \sqrt{\kappa} / \sqrt{\kappa}+\mu_{a} / \kappa+i(m \omega / l \kappa)} \text { with } \operatorname{Im}(\tau) \geq 0 \text {, we have } \\
-\Delta \Psi+\tau \Psi=\frac{q}{\sqrt{\kappa}} \text { in } \Omega, \\
\Psi+2 a \nu \cdot(\kappa \nabla \Psi)=0 \text { on } \partial \Omega .
\end{gathered}
$$

If $\tau=1$ and $q(\cdot) / \sqrt{\kappa}=\delta\left(\cdot, r_{s}\right)$ for some source point $r_{s}$, we have the following solution of (34a) as follows:

$$
\Psi(r)=R\left(r, r_{s}\right)=\frac{e^{i k\left|r-r_{s}\right|}}{4 \pi\left|r-r_{s}\right|} .
$$

For the fundamental solution with nonconstant function $\tau$, see [62].

When $\mu_{a}, \kappa, \nabla \kappa$ has upper and lower bound and $q$ is contained in $H^{-1}(\Omega)$ or a Dirac delta function, (7a), (7b), (34a), and (34b) have a unique solution $\Phi$ and $\Psi$ contained in $H^{1}(\Omega)$, respectively $[1,63]$. 
Boundary value problem (7a) and (7b) with $q(r)=$ $\delta\left(r, r_{s}\right)$ is equivalent to boundary value problem with (7a) for $q=0$ and nonzero Robin boundary condition replacing (7b). This argument can be proved using the function $H$ in [62]. Therefore, DOT is redescribed as to find the optical coefficients from Robin-to-Dirichlet map defined as a map from $H^{-1 / 2}(\partial \Omega)$ to $H^{1 / 2}(\partial \Omega)$. Using unique solvability of (7a) with Dirichlet or Neumann boundary condition replacing (7b), Robin-to-Dirichlet map is equivalent to Neumann-toDirichlet map and to Dirichlet-to-Neumann map.

4.2. Uniqueness and Nonuniqueness of DOT. The research about unique determination of the optical coefficients in DOT is rare except [61], but it is a very important issue for DOT as an inverse problem. The determination of optical coefficients $\left(\mu_{a}, \mu_{s}\right)$ in (7a) and (7b) is equivalent to the determination of $\tau$ in (34a) and (34b) when $\omega \neq 0$.

Using the relation between source-to-detector map for (7a) and (7b) and Dirichlet-to-Neumamm map for (34a), we add a comment on the result of $[42,54,61]$ and reference therein.

Case 12. $\tau$ is determined in $L^{\infty}(\Omega)$ (Theorem 5.2.2 in [54]) and $\mu_{a}, \mu_{s}^{\prime}$ is determined by comparing the real and imaginary part of $\tau$.

Case 13. $\tau$ is "almost" determined in $H_{\infty(\Omega)}^{1}$ (Theorem 5.5.3 in [54]) and $\mu_{a}, \mu_{s}^{\prime}$ is determined in a similar way for $n \geq 3$.

Case 14. Even though $\tau$ is determined, if refractive index $m$ is not known or modulation frequency $\omega=0$, we cannot determine $\mu_{a}$ and $\mu_{s}^{\prime}$, simultaneously. If $m$ is not known, we should have at least three equations and we only know at most two equations for real and imaginary part of $\tau$. And if $\omega=0$, we have no information on the imaginary part and we only have one equation. The detailed nonuniqueness example is given in [61].

In summary, if refractive index is not known or continuous light source case $\omega=0$ is used, we cannot uniquely determine the optical coefficients and if refractive index is known and frequency domain light source $\omega \neq 0$ is used, we can uniquely determine optical coefficients.

\section{Conflict of Interests}

The author declares that there is no conflict of interests regarding the publication of this paper.

\section{Acknowledgment}

This work was supported by Basic Science Research Program through the National Research Foundation of Korea (NRF) funded by the Ministry of Education (NRF2013R1A1A2010624).

\section{References}

[1] D. Gilbarg and N. S. Trudinger, Elliptic Partial Differential Equations of Second Order, Springer, 1983.

[2] H. Ammari, H. Kang, H. Lee, and M. Lim, "Enhancement of near cloaking using generalized polarization tensors vanishing structures. Part I: the conductivity problem," Communications in Mathematical Physics, vol. 317, no. 1, pp. 253-266, 2013.

[3] K. Bryan and T. Leise, "Impedance imaging, inverse problems, and Harry Potter's cloak," SIAM Review, vol. 52, no. 2, pp. 359377,2010

[4] A. Greenleaf, M. Lassas, and G. Uhlmann, "Anisotropic conductivities that cannot be detected by EIT," Physiological Measurement, vol. 24, no. 2, pp. 413-419, 2003.

[5] A. Greenleaf, M. Lassas, and G. Uhlmann, "On nonuniqueness for Calderon's inverse problem," Mathematical Research Letters, vol. 10, no. 5-6, pp. 685-693, 2003.

[6] R. V. Kohn, H. Shen, M. S. Vogelius, and M. I. Weinstein, "Cloaking via change of variables in electric impedance tomography," Inverse Problems, vol. 24, no. 1, Article ID 015016, 2008.

[7] H. Ammari, H. Kang, H. Lee, and M. Lim, "Enhancement of near-cloaking. Part II: the Helmholtz equation," Communications in Mathematical Physics, vol. 317, no. 2, pp. 485-502, 2013.

[8] R. V. Kohn, D. Onofrei, M. S. Vogelius, and M. I. Weinstein, "Cloaking via change of variables for the Helmholtz equation," Communications on Pure and Applied Mathematics, vol. 63, no. 8, pp. 973-1016, 2010.

[9] H.-M. Nguyen, "Cloaking via change of variables for the Helmholtz equation in the whole space," Communications on Pure and Applied Mathematics, vol. 63, no. 11, pp. 1505-1524, 2010.

[10] A. Greenleaf, Y. Kurylev, M. Lassas, and G. Uhlmann, "Cloaking devices, electromagnetic wormholes, and transformation optics," SIAM Review, vol. 51, no. 1, pp. 3-33, 2009.

[11] Y. Lai, J. Ng, H. Y. Chen et al., "Illusion optics: the optical transformation of an object into another object," Physical Review Letters, vol. 102, no. 25, Article ID 253902, 2009.

[12] H. Ammari, H. Kang, H. Lee, M. Lim, and S. Yu, "Enhancement of near cloaking for the full Maxwell equations," SIAM Journal on Applied Mathematics, vol. 73, no. 6, pp. 2055-2076, 2013.

[13] A. Greenleaf, Y. Kurylev, M. Lassas, and G. Uhlmann, "Approximate quantum and acoustic cloaking," Journal of Spectral Theory, vol. 1, no. 1, pp. 27-80, 2011.

[14] U. Leonhardt, "Optical conformal mapping," Science, vol. 312, pp. 1777-1780, 2006.

[15] U. Leonhardt and T. Tyc, "Broadband invisibility by nonEuclidean cloaking," Science, vol. 323, no. 5910, pp. 110-111, 2009.

[16] J. B. Pendry, D. Schurig, and D. R. Smith, "Controlling electromagnetic fields," Science, vol. 312, no. 5781, pp. 1780-1782, 2006.

[17] D. Colton and R. Kress, Inverse Acoustic and Electromagnetic Scattering Theory, vol. 93 of Applied Mathematical Sciences, Springer, Berlin, Germany, 2nd edition, 1998.

[18] V. Isakov, "On uniqueness in the inverse transmission scattering problem," Communications in Partial Differential Equations, vol. 15, no. 11, pp. 1565-1587, 1990.

[19] B. White, Developing high-density diffuse optical tomography for neuroimaging [Ph.D. thesis], Washington University in St. Louis, St. Louis, Mo, USA, 2012.

[20] Q. Zang, T. J. Brukilacchio, A. Li et al., "Coregistered tomogrpahic X-ray and optical breast imaging: initial results," Journal of Biomedical Optics, vol. 10, no. 2, Article ID 024033, 2005. 
[21] T. Durduran, R. Choe, G. Yu et al., "Diffuse optical measurement of blood flow in breast tumors," Optics Letters, vol. 30, no. 21, pp. 2915-2917, 2005.

[22] V. Ntziachristos, A. G. Yodh, M. Schnall, and B. Chance, "Concurrent MRI and diffuse optical tomography of breast after indocyanine green enhancement," Proceedings of the National Academy of Sciences of the United States of America, vol. 97, no. 6, pp. 2767-2772, 2000.

[23] J. P. Culver, A. M. Siegel, M. A. Franceschini, J. B. Mandeville, and D. A. Boas, "Evidence that cerebral blood volume can provide brain activation maps with better spatial resolution than deoxygenated hemoglobin," NeuroImage, vol. 27, no. 4, pp. 947-959, 2005.

[24] M. A. Franceschini and D. A. Boas, "Noninvasive measurement of neuronal activity with near-infrared optical imaging," $\mathrm{Neu}$ roImage, vol. 21, no. 1, pp. 372-386, 2004.

[25] R. Sitaram, H. Zhang, C. Guan et al., "Temporal classification of multichannel near-infrared spectroscopy signals of motor imagery for developing a brain-computer interface," NeuroImage, vol. 34, no. 4, pp. 1416-1427, 2007.

[26] S. M. Coyle, T. E. Ward, and C. M. Markham, "Braincomputer interface using a simplified functional near-infrared spectroscopy system," Journal of Neural Engineering, vol. 4, no. 3, pp. 219-226, 2007.

[27] E. Watanabe, A. Maki, F. Kawaguchi, Y. Yamashita, H. Koizumi, and Y. Mayanagi, "Noninvasive cerebral blood volume measurement during seizures using multichannel near infrared spectroscopic topography," Journal of Biomedical Optics, vol. 5, no. 3, pp. 287-290, 2000.

[28] K. Buchheim, H. Obrig, W. V. Pannwitz et al., "Decrease in haemoglobin oxygenation during absence seizures in adult humans," Neuroscience Letters, vol. 354, no. 2, pp. 119-122, 2004.

[29] G. Taga, Y. Konishi, A. Maki, T. Tachibana, M. Fujiwara, and H. Koizumi, "Spontaneous oscillation of oxy- and deoxyhemoglobin changes with a phase difference throughout the occipital cortex of newborn infants observed using noninvasive optical topography," Neuroscience Letters, vol. 282, no. 1-2, pp. 101-104, 2000.

[30] T. Austin, A. P. Gibson, G. Branco et al., "Three dimensional optical imaging of blood volume and oxygenation in the neonatal brain," NeuroImage, vol. 31, no. 4, pp. 1426-1433, 2006.

[31] Z. Yuan, Q. Zhang, E. Sobel, and H. Jiang, “Three-dimensional diffuse optical tomography of osteoarthritis: initial results in the finger joints," Journal of Biomedical Optics, vol. 12, no. 3, Article ID 034001, 2007.

[32] A. M. Siegel, J. P. Culver, J. B. Mandeville, and D. A. Boas, "Temporal comparison of functional brain imaging with diffuse optical tomography and fMRI during rat forepaw stimulation," Physics in Medicine and Biology, vol. 48, no. 10, pp. 1391-1403, 2003.

[33] J. P. Culver, T. Durduran, D. Furuya, C. Cheung, J. H. Greenberg, and A. G. Yodh, "ffuse optical tomography of cerebral blood flow, oxygenation, and metabolism in rat during focal ischemia," Journal of Cerebral Blood Flow \& Metabolism, vol. 23, no. 8, pp. 911-924, 2003.

[34] B. Barceló, E. Fabes, and J. K. Seo, “The inverse conductivity problem with one measurement: uniqueness for convex polyhedra," Proceedings of the American Mathematical Society, vol. 122, no. 1, pp. 183-189, 1994.

[35] A. Friedman and V. Isakov, "On the uniqueness in the inverse conductivity problem with one measurement," Indiana University Mathematics Journal, vol. 38, no. 3, pp. 563-579, 1989.
[36] H. Kang and J. K. Seo, "The layer potential technique for the inverse conductivity problem," Inverse Problems, vol. 12, no. 3, pp. 267-278, 1996.

[37] K. Astala and L. Päivärinta, "Calderon 's inverse conductivity problem in the plane," Annals of Mathematics, vol. 163, no. 1, pp. 265-299, 2006.

[38] R. M. Brown and G. A. Uhlmann, "Uniqueness in the inverse conductivity problem for nonsmooth conductivities in two dimensions," Communications in Partial Differential Equations, vol. 22, no. 5-6, pp. 1009-1027, 1997.

[39] A. I. Nachman, "Global uniqueness for a two-dimensional inverse boundary value problem," Annals of Mathematics. Second Series, vol. 143, no. 1, pp. 71-96, 1996.

[40] G. Alessandrini, "Stable determination of conductivity by boundary measurements," Applicable Analysis, vol. 27, no. 1-3, pp. 153-172, 1988.

[41] A. I. Nachmann, "Reconstruction from boundary measurements," Annals of Mathematics, vol. 128, pp. 531-576, 1988.

[42] J. Sylvester and G. Uhlmann, "A global uniqueness theorem for an inverse boundary value problem," Annals of Mathematics, vol. 125, no. 1, pp. 153-169, 1987.

[43] J. Sylvester and G. Uhlmann, "Inverse boundary value problems at the boundary-continuous dependence," Communications on Pure and Applied Mathematics, vol. 41, no. 2, pp. 197-219, 1988.

[44] V. Isakov, "On uniqueness of recovery of a discontinuous conductivity coefficient," Communications on Pure and Applied Mathematics, vol. 41, no. 7, pp. 865-877, 1988.

[45] M. Ikehata, "Identification of the curve of discontinuity of the determinant of the anisotropic conductivity," Journal of Inverse and Ill-Posed Problems, vol. 8, no. 3, pp. 273-285, 2000.

[46] K. Kwon and D. Sheen, "Anisotropic inverse conductivity and scattering problems," Inverse Problems, vol. 18, no. 3, pp. 745756, 2002.

[47] R. Kohn and M. Vogelius, "Determining conductivity by boundary measurements," Communications on Pure and Applied Mathematics, vol. 37, no. 3, pp. 289-298, 1984.

[48] J. Sylvester, "An anisotropic inverse boundary value problem," Communications on Pure and Applied Mathematics, vol. 43, no. 2, pp. 201-232, 1990.

[49] Z. Sun and G. Uhlmann, "Anisotropic inverse problems in two dimensions," Inverse Problems, vol. 19, no. 5, pp. 1001-1010, 2003.

[50] K. Astala, L. Päivärinta, and M. Lassas, "Calderóns' inverse problem for anisotropic conductivity in the plane," Communications in Partial Differential Equations, vol. 30, no. 1-3, pp. 207224, 2005.

[51] M. Lassas and G. Uhlmann, "On determining a riemannian manifold from the cirichlet-to-neumann map," Annales Scientifiques de l'École Normale Supérieure, vol. 34, no. 5, pp. 771-787, 2001.

[52] J. M. Lee and G. Uhlmann, "Determining anisotropic realanalytic conductivities by boundary measurements," Communications on Pure and Applied Mathematics, vol. 42, no. 8, pp. 1097-1112, 1989.

[53] K. Kwon, "Identification of anisotropic anomalous region in inverse problems," Inverse Problems, vol. 20, no. 4, pp. 1117-1136, 2004.

[54] V. Isakov, Inverse Problems for Partial Differential Equations, vol. 127 of Applied Mathematical Sciences, Springer, Berlin, Germany, 2nd edition, 2006. 
[55] A. Kirsch and R. Kress, "Uniqueness in inverse obstacle scattering," Inverse Problems, vol. 9, no. 2, pp. 285-299, 1993.

[56] G. Eskin, "Inverse scattering problem in anisotropic media," Communications in Mathematical Physics, vol. 199, no. 2, pp. 471-491, 1998.

[57] F. Gylys-Colwell, "An inverse problem for the Helmholtz equation," Inverse Problems, vol. 12, no. 2, pp. 139-156, 1996.

[58] M. Piana, "On uniqueness for anisotropic inhomogeneous inverse scattering problems," Inverse Problems, vol. 14, no. 6, pp. 1565-1579, 1998.

[59] R. Potthast, "Electromagnetic scattering from an orthotropic medium," Journal of Integral Equations and Applications, vol. 11, no. 2, pp. 197-215, 1999.

[60] D. Sheen and D. Shepelsky, "Inverse scattering problem for a stratified anisotropic slab," Inverse Problems, vol. 15, no. 2, pp. 499-514, 1999.

[61] S. R. Arridge and W. R. B. Lionheart, "Nonuniqueness in diffusion-based optical tomography," Optics Letters, vol. 23, no. 11, pp. 882-884, 1998.

[62] C. Miranda, Partial Differential Equations of Elliptic Type, Springer, 1970.

[63] K. Kwon and B. Yazıcı, "Born expansion and Fréchet derivatives in nonlinear diffuse optical tomography," Computers \& Mathematics with Applications, vol. 59, no. 11, pp. 3377-3397, 2010. 


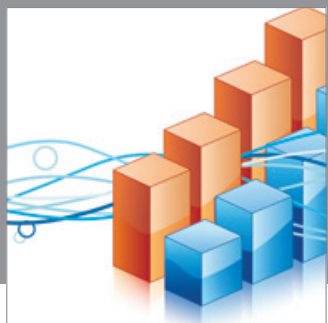

Advances in

Operations Research

mansans

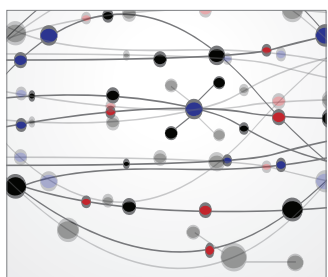

The Scientific World Journal
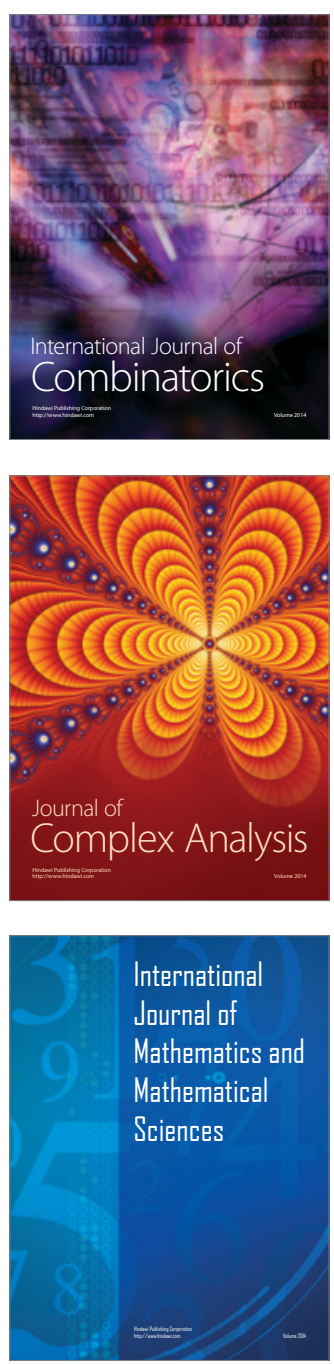
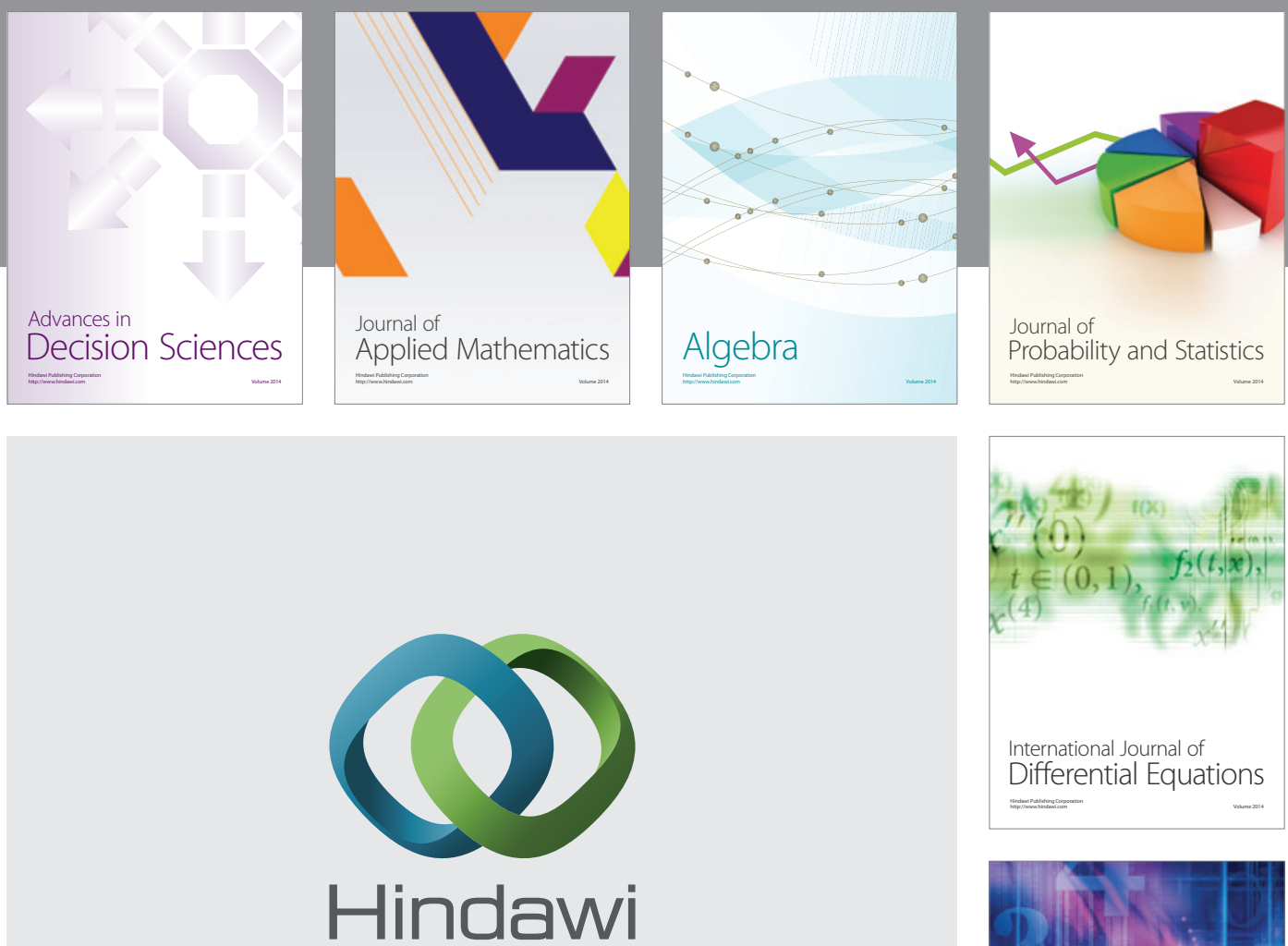

Submit your manuscripts at http://www.hindawi.com
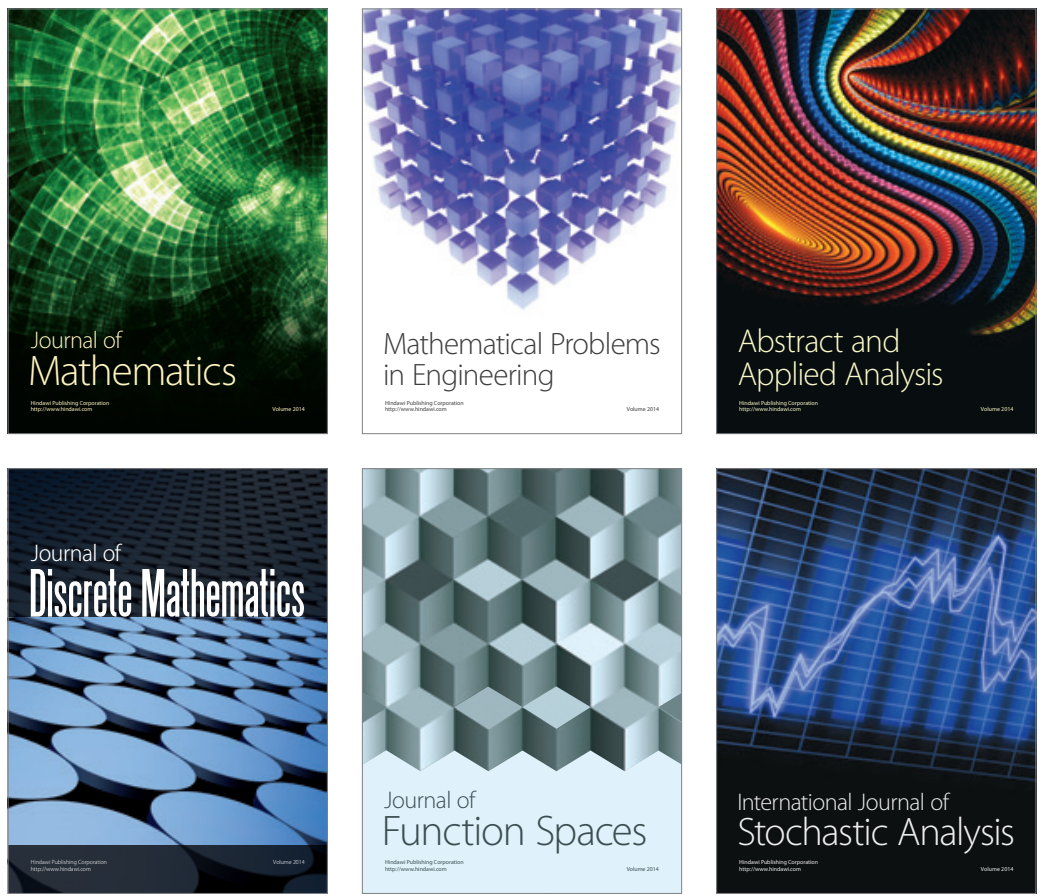

Journal of

Function Spaces

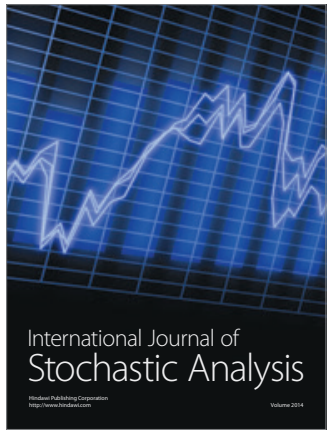

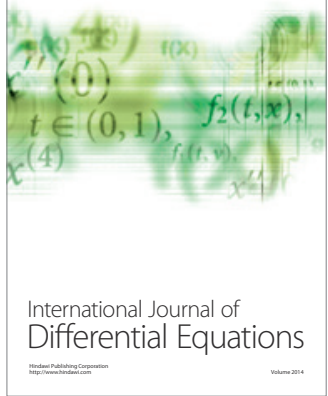
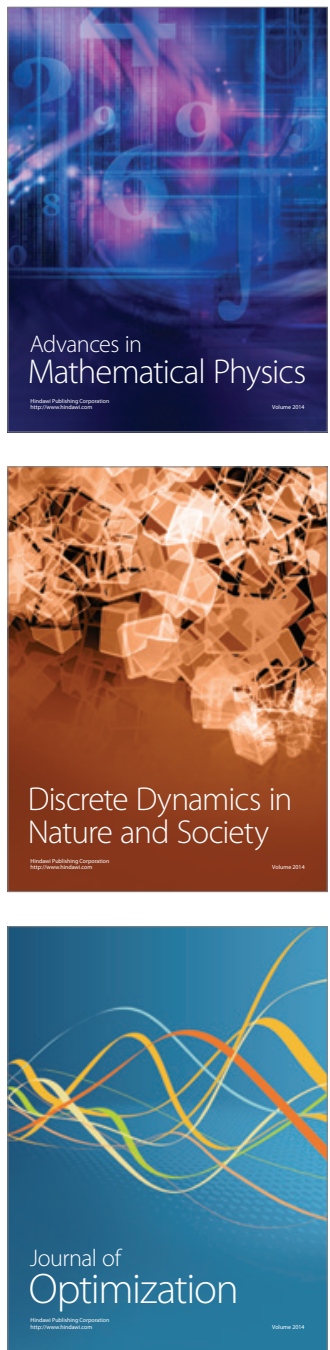